\title{
Análise epidemiológica dos resultados de exames parasitológicos de protozooses intestinais no município de Teresina - PI
} Epidemiological analysis of the results of parasitological exams of intestinal protozooses in
the city of Teresina - PI

Análisis epidemiológico de los resultados de los exámenes parasitológicos de protozoos intestinales en la ciudad de Teresina - PI

Matheus Rodrigues Nogueira ${ }^{1 *}$, Augusto César Evelin Rodrigues ${ }^{1}$, André Neiva Pinheiro Correia ${ }^{1}$, Charles Gonçalves Barroso de Sousa ${ }^{1}$, Julie Rocha Porto ${ }^{1}$, Maria Luisa Oliveira Rocha ${ }^{1}$, Yasmim Fernandes Mota da Rocha'.

\section{RESUMO}

Objetivo: Analisar a incidência de protozooses intestinais dos resultados dos exames de fezes e mapear os resultados positivos, segundo espécie do protozoário, sexo, idade e zona acometida dos pacientes no município de Teresina-PI. Métodos: Tratou-se de um estudo documental com abordagem quantitativa de natureza descritiva e retrospectiva, no qual foi contemplado a distribuição temporal dos casos de protozooses intestinais na população do município de Teresina-PI, no período de janeiro a setembro de 2018 , feita através da coleta dos resultados exames de fezes de um laboratório público municipal de referência. Resultados: Observou-se que, de um total de 23694 exames parasitológicos de fezes, 8,43\% foram positivos para protozoários, sendo sexo feminino o mais acometido (61,76\%). Quanto a zona mais afetada, 67,27\% eram de zona urbana. Dentre as faixas etárias, a prevalência foi maior entre 1 a 10 anos $(25,58 \%)$ e 11 a 20 anos $(19,72 \%)$. Já com relação as espécies de protozoários mais prevalentes, três representam $96 \%$ dos casos acometidos que são: a Entamoeba coli (33,93\%), a Entamoeba histolytica (36,69\%) e a Giardia lamblia (25,53\%). Conclusão: Conclui-se que a protozoose intestinal constitui um problema de saúde pública e são endêmicas em países em desenvolvimento, afetando, principalmente, a população com piores condições socioeconômicas.

Palavras-chave: Epidemiologia, Saneamento básico, Saúde pública, Infecções por protozoários.

\begin{abstract}
Objective: To analyze the incidence of intestinal protozooses from the results of stool examinations of patients from Teresina-PI and map the positive results, according to species, sex, age and affected area. Methods: It was a documentary study with a quantitative approach of a descriptive and retrospective nature, in which the temporal distribution of cases of intestinal protozooses in the population in Teresina-PI, from January to September 2018, was contemplated through the collection stool exam results from a public municipal reference laboratory. Results: It was observed that, of a total of 23694 parasitological examinations of feces, $8.43 \%$ were positive for protozoa, with females being the most affected (61.76\%). As for the most affected area, $67.27 \%$ were from urban areas. Among the age groups, the prevalence was higher between 1 to 10 years $(25.58 \%)$ and 11 to 20 years (19.72\%). Regarding the most prevalent species of protozoa, three represent 96\% of the affected cases, which are: Entamoeba coli (33.93\%), Entamoeba histolytica (36.69\%) and Giardia lamblia (25.53\%). Conclusion: It is concluded that intestinal protozoosis is a public health problem and is endemic in developing countries, affecting mainly the population with the worst socioeconomic conditions.
\end{abstract}

Key words: Epidemiology, Basic sanitation, Public health, Protozoan infections.

${ }^{1}$ Centro Universitário UniFacid Wyden, Teresina - PI. * E-mail: matheus.nogueira@hotmail.com 


\section{RESUMEN}

Objetivo: Analizar la incidencia de protozoos intestinales a partir de los resultados de exámenes de heces de pacientes de Teresina-PI y mapear los resultados positivos, según especie, sexo, edad y área afectada. Métodos: Fue un estudio documental con abordaje cuantitativo de carácter descriptivo y retrospectivo, en el que se realizó la distribución temporal de casos de protozoos intestinales en la población de Teresina-PI, de enero a septiembre de 2018 , a través de la colección. resultados del examen de heces de un laboratorio municipal público de referencia. Resultados: Se observó que, de un total de 23694 exámenes parasitológicos de heces, el $8.43 \%$ fueron positivos para protozoos, siendo las hembras las más afectadas (61.76\%). En cuanto a la zona más afectada, el $67,27 \%$ eran de zonas urbanas. Entre los grupos de edad, la prevalencia fue mayor entre 1 a 10 años (25,58\%) y 11 a 20 años (19,72\%). En cuanto a las especies de protozoos más prevalentes, tres representan el $96 \%$ de los casos afectados, que son: Entamoeba coli (33,93\%), Entamoeba histolytica (36,69\%) y Giardia lamblia (25,53\%). Conclusión: Se concluye que la protozoosis intestinal es un problema de salud pública y es endémico en los países en desarrollo, afectando principalmente a la población con peores condiciones socioeconómicas.

Palabras clave: Epidemiología, Saneamiento básico, Salud pública, Infecciones por protozoos.

\section{INTRODUÇÃO}

Parasitoses intestinais são enfermidades causadas por helmintos ou por protozoários, sendo comuns ao redor do mundo, mais frequentes na África, Ásia e America Latina. A prevalência dessas infecções por parasitas intestinais é um dos melhores indicadores do status socioeconômico de uma população e pode estar associada a diversos determinantes, como contaminação do solo, da água e de alimentos, instalações sanitárias inadequadas, deficiência no saneamento básico, pobreza, contato com animais, além da idade do hospedeiro e do tipo de parasito infectante (BELO VS, et al., 2012).

Estudos mostraram que as principais enteroparasitoses estão entre as 20 doenças mais frequentes e entre os maiores índices de mortalidade nos continentes africano, asiático e latino-americano. No que diz a respeito aos protozoários, estima-se que afetem entre 200 a 400 milhões de pessoas no planeta, sendo Entamoeba histolytica e Giardia intestinalis as espécies mais prevalentes (MAGALHÃES RF, 2013; MAIA CVA e HASSUM IC, 2016).

Suas consequências (sintomas) mais frequentes, tais como: dores abdominais, diarreia, gases, falta de apetite, perda de peso, náuseas, vômitos, tosse, febre, falta de ar, anemia, dentre outros, podem causar graves deficiências nutricionais. Constitui-se como um grave problema de saúde pública, tais infecções são alguns dos principais fatores debilitantes da população, associando-se, frequentemente, a quadros de diarreia crônica e desnutrição, comprometendo, assim, o desenvolvimento físico e intelectual, particularmente das faixas etárias mais jovens da população (BORGES WF, et al., 2011; FREITAS MRS, et al., 2015).

Embora afetem uma ampla distribuição geográfica, a intensidade dessas infecções é influenciada por fatores ambientais, domésticos, nutricionais e outras características ligadas a pobreza. O principal fator de risco associado a essa enfermidade é a falta de acesso a água canalizada. Conforme a Organização Mundial de Saúde (OMS), em 2017, a deficiência de saneamento básico acometeu 2,4 bilhões de pessoas e mais de 660 milhões consumiram água sem o tratamento adequado (IGNACIO CF, et al., 2017; CRUZ JS, et al., 2020).

Com isso, os países subdesenvolvidos são os mais afetados, já que parte da população vive em situações precárias, sem acesso à distribuição de água potável, sem coleta e tratamento de esgoto, o que favorece a transmissão dessa enfermidade, uma vez que a água não tratada pode ser um considerável propagador de cistos de protozoários. No Brasil, somente cerca de $60 \%$ da população tem acesso à água encanada e $45 \%$ possuem acesso ao saneamento básico, ou seja, mais da metade das pessoas estão expostas ao principal fator de risco (MONTEIRO ACS, et al., 2017; SILVA AMB, et al., 2014).

A ineficiência em fornecer estrutura de moradia, de saneamento básico e de água encanada de forma adequada nas regiões é um dos principais determinantes para a população adquirir as protozooses intestinais, 
pois as parasitoses podem ser transmitidas de três formas: por meio da contaminação oral (a partir da ingestão dos ovos ou cistos dos parasitas nos alimentos ou na água contaminada), por meio da penetração de larvas na pele e nas mucosas (quando entramos em contato com as larvas de determinados parasitas encontradas no solo) e por meio do contato com fezes de humanos não saudáveis (VIEIRA PB, et al., 2012; BRAGAGNOLLO GR, et al., 2018).

Um pequeno grupo de protozoários tem impacto significativo na saúde pública, sendo composto pelos organismos Entamoeba histolytica e Giardia lamblia (também conhecida como Giardia intestinalis e Giardia duodenalis), capazes de levar prejuízo no status nutricional do hospedeiro, afetando, principalmente, a absorção e o metabolismo de macro e micronutrientes, pois podem levar à diarreia crônica, anemia por deficiência de ferro e subnutrição, resultando no retardo do crescimento, no comprometimento do desenvolvimento neuropsicomotor, na função imunológica, na capacidade produtiva e na capacidade intelectual (SILVA JVL, et al., 2016).

Para o diagnóstico das protozooses é utilizado o exame parasitológico de fezes (EPF), que possui alta especificidade. Entretanto, apresenta uma baixa sensibilidade, ou seja, nem sempre é capaz de identificar a presença de parasita nas fezes. Para contornar essa sensibilidade, coleta-se um maior número de amostras e aumenta-se o intervalo de tempo entre as coletas. Todavia, na prática médica, alguns profissionais preferem o tratamento empírico baseado na clínica e nos fatores epidemiológicos da região, sendo os derivados nitroimidazólicos (metronidazol ou secnidazol) o grupo de escolha para as protozooses intestinais (giardíase e amebíase), já que tem um baixo custo, sendo distribuído gratuitamente pelo Sistema Único de Saúde (SUS) e tem uma eficácia clínica de até $93 \%$ para ambos os protozoários (ANDRADE EC, et al., 2010; KAPPADOGA S, et al., 2011).

Ainda que, nas últimas décadas, o Brasil tenha passado por modificações que melhoraram a qualidade de vida de sua população, as parasitoses intestinais ainda são endêmicas em diversas áreas do país, constituindo um problema relevante de Saúde Pública. Os dados do Instituto Brasileiro de Geografia e Estatística (IBGE), em 2010, mostram que o munícipio de Teresina-PI não oferecia condições básicas de saneamento, já que somente $69 \%$ dos domicílios urbanos apresentavam esgotamento sanitário adequado. Na zona rural, esses números são mais discrepantes, pois apenas $8,3 \%$ das moradias detinham as condições que são consideradas básicas (IBGE, 2010; BELO VS, et al., 2012; CARCARÁ, MSM, et al., 2019).

Dessa maneira, a principal profilaxia para essas protozooses intestinais seria o investimento na melhoria do saneamento básico, do abastecimento hídrico, das condições de habitação e da conscientização da população sobre higiene pessoal e coletiva, em todas as regiões. Portanto, através deste estudo, será possível analisar a incidência de protozooses intestinais dos resultados dos exames de fezes e mapear os resultados positivos, segundo a espécie do protozoário, o sexo, a idade e a zona acometida dos pacientes no município de Teresina - PI. Os dados colhidos deverão, assim, permitir a suplementação das medidas de prevenção e controle da doença nas áreas mais prevalentes.

\section{MÉTODOS}

Tratou-se de um estudo do tipo documental, descritivo, com abordagem de natureza quantitativa, retrospectiva, caracterizando uma análise de saúde, na qual foi abordada a distribuição temporal dos casos de parasitoses intestinais na população do município em Teresina-Piauí, no período de Janeiro a Setembro de 2018.

A pesquisa apresenta natureza descritiva por ter como propósito a observação, descrição, exploração, classificação e interpretação dos aspectos de fatos ou fenômenos, buscando a frequência, as características, a relação e associação entre variáveis. Além disso, possui uma abordagem quantitativa, uma vez que há coleta sistemática de informações que podem ser quantificadas em condições de controle, além da análise das informações através de procedimentos estatísticos (DYNIEWICZ AM, 2009).

Os dados foram coletados dos resultados exames de fezes dos pacientes de um laboratório público municipal de referência e os participantes do estudo foram todos os casos de pacientes com protozooses intestinais em Teresina-Piauí, no período de Janeiro a Setembro de 2018. 
Foi utilizado como critério de inclusão: ser caso diagnosticado de protozooses intestinais, ser residente em Teresina-PI no período de Janeiro a Setembro de 2018 e, por fim, ter sido diagnosticado através dos exames parasitológicos de fezes por um laboratório público municipal de referência. Como critério de exclusão foi utilizado ser paciente morador de outro município do Piauí ou de outro estado da federação.

Os dados foram colhidos de planilhas digitais, dos resultados dos exames dos pacientes de um laboratório público municipal de referência e digitado em planilha do programa EXCEL-2019, no período de Janeiro a Setembro de 2019, buscando-se levantar a seguintes variáveis de interesse do estudo: dados sociodemográficos (sexo, idade e zona de residência dos pacientes) e dados dos pacientes (espécie dos parasitas).

Os dados foram organizados em planilhas do programa EXCEL-2019 e analisadas através de frequência absoluta e relativa e através de estatística básica na base de 100. Os dados foram reunidos através de tabelas para melhorar compreensão.

A pesquisa foi realizada conforme as normas da Resolução 466/2012 do Conselho Nacional de Saúde. Para a realização da coleta de dados, o projeto teve a aprovação do Comitê de Ética em Pesquisa (CEP) da Faculdade Integral Diferencial (FACID|Wyden), parecer n 4.324.954, e da Fundação Municipal de Saúde, responsável pela realização dos exames.

Dessa forma, esse trabalho torna-se relevante ao fornecer um estudo com caráter retrospectivo, descritivo e exploratório, com dados secundários, sobre a incidência de protozooses intestinais em pacientes do município de Teresina-PI, bem como identificar esses parasitas segundo a espécie. Além disso, visa ampliar o conhecimento sobre o tema e servir como base para pesquisas futuras.

\section{RESULTADOS}

A análise das protozooses mais prevalentes e dos aspectos sociodemográficos foram obtidas por meio do levantamento de dados do laboratório público municipal de referência, que utilizou como base os resultados dos exames parasitológicos de fezes (EPF) solicitados por médicos, entre o período de Janeiro a Setembro de 2018, no município de Teresina - PI.

Observou-se que cerca de $8,43 \%$ dos exames parasitológicos de fezes foram positivos para protozoários, de um total de 23694 realizados. Dentre eles, ocorreu uma incidência maior de Entamoeba histolytica com $36,69 \%$, seguido de Entamoeba coli com 33,93\% e de Giardia intestinalis com 25,53\% (Tabela 1). Outros protozoários como Endolimax nana, lodamoeba butschlii e Balantidium coli também foram positivos na amostragem, entretanto, com uma menor prevalência.

Tabela 1 - Distribuição dos protozoários nos exames parasitológicos de fezes positivos, $\mathrm{n}=$ 1998. Teresina $-\mathrm{PI}, 2018$.

\begin{tabular}{lcc}
\hline Variável & N & $\%$ \\
\hline Espécies de protozoários & & \\
\hline Entamoeba histolytica & 733 & 36,69 \\
Entamoeba coli & 678 & 33,93 \\
Giardia intestinalis & 510 & 25,53 \\
Endolimax nana & 56 & 2,80 \\
lodamoeba butschlii & 20 & 1,00 \\
Balantidium coli & 1 & 0,05 \\
\hline Total & $\mathbf{1 9 9 8}$ & $\mathbf{1 0 0}$
\end{tabular}

Fonte: Nogueira MR, et al., 2021.

Os resultados da distribuição sociodemográfica dos exames positivos demonstraram uma maior prevalência de casos positivos em mulheres $(61,76 \%)$ (Tabela 2$)$. Em relação à faixa etária da amostra total da população, dois intervalos evidenciaram maior proporção, sendo eles: 0 a 10 anos $(25,58 \%)$ e 11 a 20 anos (19,72\%). Por fim, os dados mostraram que $67,27 \%$ dos pacientes residiam na zona urbana. 
Tabela 2 - Distribuição dos casos de protozooses positivos classificados por sexo, faixa etária e zona que reside, $\mathrm{n}=1998$. Teresina - PI, 2018.

\begin{tabular}{lcc}
\hline Variáveis & N & $\%$ \\
\hline Sexo & & \\
\hline Masculino & 764 & 38,24 \\
Feminino & 1234 & 61,76 \\
Faixa etária & & \\
\hline De 0 a 10 anos & 511 & 25,58 \\
De 11 a 20 anos & 394 & 19,72 \\
De 20 a 30 anos & 223 & 11,16 \\
De 30 a 40 anos & 233 & 11,66 \\
De 41 a 50 anos & 220 & 11,01 \\
De 51 a 60 anos & 194 & 9,71 \\
Acima de 61 anos & 195 & 9,76 \\
Não informado & 28 & 1,40 \\
\hline Zona de Residência & & \\
\hline Urbana & 1344 & 67,27 \\
Rural & 87 & 4,35 \\
Não informado & 567 & 28,38 \\
\hline Total & $\mathbf{1 9 9 8}$ & $\mathbf{1 0 0}$ \\
\hline
\end{tabular}

Fonte: Nogueira MR, et al., 2021.

Entre os exames positivos para as espécies mais predominantes, E. histolytica, E. coli e G. intestinalis, os dados da distribuição sociodemográfica foram similares, sendo o sexo feminino o mais acometido e a zona urbana a área mais afetada do munícipio (Tabela 3, Tabela 4 e Tabela 5). No que diz a respeito ao intervalo das idades, a proporção variou entre cada espécie, no qual 0 a 10 anos foi o período mais acometido na $E$. histolytica $(31,92 \%)$ e na $G$. intestinalis $(26,47 \%)$. Porém, a E. coli acometeu mais a faixa entre 11 a 20 anos $(21,98 \%)$, apesar da parcela de 0 a 10 anos ser semelhante $(18,73 \%)$.

Tabela 3 - Distribuição dos casos de E. histolytica positivos classificados por sexo, faixa etária e zona que reside, $\mathrm{n}=$ 733. Teresina - PI, 2018.

\begin{tabular}{ccc}
\hline Variáveis & N & $\%$ \\
\hline Sexo & & \\
\hline Masculino & 293 & 39,97 \\
Feminino & 440 & 60,03 \\
Faixa etária & 234 & 31,92 \\
\hline De 0 a 10 anos & 124 & 16,92 \\
De 11 a 20 anos & 76 & 10,37 \\
De 20 a 30 anos & 83 & 11,32 \\
De 30 a 40 anos & 74 & 10,10 \\
De 41 a 50 anos & 66 & 9,00 \\
De 51 a 60 anos & 68 & 9,28 \\
Acima de 61 anos & 8 & 1,09 \\
Não informado & & \\
\hline Zona de Residência & 503 & 68,62 \\
\hline Urbana & 40 & 5,46 \\
Rural & 190 & 25,92 \\
Não informado & $\mathbf{7 3 3}$ & $\mathbf{1 0 0}$ \\
\hline Total & &
\end{tabular}

Fonte: Nogueira MR, et al., 2021. 
Tabela 4 - Distribuição dos casos de E. coli positivos classificados por sexo, faixa etária e zona que reside, $\mathrm{n}=678$. Teresina - $\mathrm{Pl}, 2018$.

\begin{tabular}{lcc}
\hline Variáveis & N & $\%$ \\
\hline Sexo & & \\
\hline Masculino & 262 & 38,64 \\
Feminino & 416 & 61,36 \\
Faixa etária & & \\
\hline De 0 a 10 anos & 127 & 18,73 \\
De 11 a 20 anos & 149 & 21,98 \\
De 20 a 30 anos & 76 & 11,21 \\
De 30 a 40 anos & 76 & 11,21 \\
De 41 a 50 anos & 80 & 11,80 \\
De 51 a 60 anos & 85 & 12,54 \\
Acima de 61 anos & 80 & 11,80 \\
Não informado & 5 & 0,74 \\
\hline Zona de Residência & & \\
\hline Urbana & 385 & 56,78 \\
Rural & 35 & 5,16 \\
Não informado & 258 & 38,05 \\
\hline Total & $\mathbf{6 7 8}$ & $\mathbf{1 0 0}$
\end{tabular}

Fonte: Nogueira MR, et al., 2021.

Tabela 5 - Distribuição dos casos de G. intestinalis positivos classificados por sexo, faixa etária e zona que reside, $\mathrm{n}=510$. Teresina - PI, 2018.

\begin{tabular}{lcc}
\hline Variáveis & N & $\%$ \\
\hline Sexo & & \\
\hline Masculino & 188 & 36,86 \\
Feminino & 322 & 63,13 \\
\hline Faixa etária & & \\
\hline De 0 a 10 anos & 135 & 26,47 \\
De 11 a 20 anos & 103 & 20,20 \\
De 20 a 30 anos & 64 & 12,55 \\
De 30 a 40 anos & 63 & 12,35 \\
De 41 a 50 anos & 54 & 11,01 \\
De 51 a 60 anos & 38 & 7,45 \\
Acima de 61 anos & 40 & 7,84 \\
Não informado & 13 & 2,55 \\
\hline Zona de Residência & & \\
\hline Urbana & 394 & 27,25 \\
Rural & 12 & 2,35 \\
Não informado & 104 & $\mathbf{1 0 0}$
\end{tabular}

Fonte: Nogueira MR, et al., 2021.

\section{DISCUSSÃO}

Estima-se que infecções intestinais causadas por helmintos e protozoários afetem cerca de 3,5 bilhões de pessoas no mundo, causando enfermidades em, aproximadamente, 450 milhões de pacientes ao redor do mundo, a maior parte destas em crianças, segundo a Organização Mundial de Saúde. No Brasil, essas infecções ocorrem tanto na área rural quanto na área urbana e em diferentes faixas etárias (BELO VS, et al., 2012; SANTOS AS e MERLINI LS, 2010). 
A prevalência de infecções por parasitoses intestinais é um dos melhores indicadores do status socioeconômico de uma população e pode estar associada a diversos determinantes, como instalações sanitárias inadequadas, poluição fecal da água e de alimentos consumidos, fatores socioculturais, contato com animais, ausência de saneamento básico, além da idade do hospedeiro e do tipo de parasito infectante (GAMBOA MI, et al., 2003).

Quanto às espécies identificadas, o Entamoeba histolytica foi o protozoário patogênico mais frequente (36,69\%), seguido pela Entamoeba coli (33,93\%), corroborando com alguns estudos da literatura regional em que mostra que o complexo Entamoeba histolytica/díspar foi o mais prevalente em análise no Hospital Universitário de João Pessoa - PB e no estado do Maranhão na faixa etária de 11 a 30 anos (DANTAS SH, et al., 2019; SILVA FS, et al., 2010).

A amebíase, até 1993, era descrita como um único protozoário que infectava cerca de $10 \%$ da população mundial. Entretanto, ela foi dividida, posteriormente, em duas espécies: $E$. histolytica que é patogênico, podendo causar a forma invasiva e suas complicações; e $E$. díspar que não é patogênico para os seres humanos, sendo a E. coli um protozoário pertencente desse gênero (SILVA MTN, et al., 2014). A prevalência da infecção por E. histolytica é variável entre as regiões do Brasil, sendo encontrado com bastante frequência na região Nordeste do Brasil, justamente devido as diferenças socioeconômicas e do saneamento básico (DANTAS, SH, et al., 2019).

O presente estudo mostra que a Giardia intestinalis foi a $3^{\text {a }}$ mais prevalente com $25,53 \%$, sendo que a frequência da giardíase varia a depender do país, variando entre 11 a 30\% (MBUH JV, et al., 2010). No Brasil, a protozoose varia dependendo da região, da pobreza e da idade da população estudada (IGNACIO CF, et al., 2017). Estudo realizado no Maranhão corrobora com o atual, sendo o segundo protozoário patogênico mais frequente na população atrás da E. histolytica (SILVA FS, et al., 2010).

Em países em desenvolvimento, as infecções por G. intestinalis são particularmente comuns entre as crianças, sobretudo, na faixa etária entre 0 a 12 anos, reforçando o resultado encontrado no presente estudo, na qual a faixa etária de 0 a 10 anos $(26,47 \%)$ foi a mais acometida pela protozoose (VIVANCOS V, et al., 2018). A maioria dessas infecções são assintomáticas, contudo, representam uma séria ameaça à saúde e ao desenvolvimento das crianças, podendo gerar atraso no desenvolvimento neuropsicomotor (BORGES WF, et al., 2011).

Foram encontradas 56 amostras positivas para Endolimax nana (2,8\%) que, junto com a Entamoeba coli fazem parte do grupo dos protozoários comensais, representando cerca de $36,73 \%$ das amostras positivas. A E. coli, uma ameba não patogênica, é transmitida de forma semelhante a outras protozooses como $E$. histolytica e G. intestinalis, portanto esses resultados positivos nos Exames Parasitológicos de Fezes (EPF) demostram que a população é exposta devido às condições sanitárias (CHAVES ACP, et al., 2010). A importância epidemiológica de reportar protozoários comensais nos EPF é pertinente, pois demonstra a inadequação sanitária da região, já que existe a contaminação do solo e dos recursos hídricos, o que se torna propício para disseminação de protozooses intestinais (DANTAS SH, et al., 2019; LOPES LM, et al., 2010).

Entre os protozoários menos prevalentes encontrados na pesquisa, sendo eles lodamoeba butschlii e Balantidium coli, podemos observar que, em outras regiões, a ocorrência deles segue o padrão desse estudo. Em 410 amostras fecais de crianças residentes da Zona Rural de Ipirá - BA, apenas 3,4\% apresentavam $I$. butschlii. Já sobre o Balantidium coli, patógeno que causa a balantidiose, existem poucos estudos na literatura nacional que mostram a prevalência desse protozoário. Porém, de acordo literatura internacional, entre as doenças causadas pela veiculação hídrica na América do Norte e na Europa, apenas 0,3\% foram causadas pela $B$. coli, o que valida a baixa prevalência dessa protozoose na pesquisa atual, com apenas 1 amostra positiva (KARANIS P, et al., 2007; SANTOS-JUNIOR GO, et al., 2006).

Ao se observar os resultados da distribuição sociodemográfica dos pacientes analisados diante à prevalência de protozooses intestinais, a faixa etária mais acometida, com um total de 511 pessoas, foi de 0 a 10 anos de idade (25,58\%), fase onde as crianças descobrem as texturas dos alimentos com as mãos e acabam levando-as até a boca com maior frequência. Além disso, é na infância onde ainda se aprende sobre 
a correta lavagem e higienização das mãos em momentos oportunos e cruciais, como antes das refeições e após as eliminações fisiológicas. O segundo intervalo com mais infectados foi de 11 a 20 anos, com um total de 394 pessoas, perfazendo $19,72 \%$ dos analisados, o que demonstra ser um grupo com imunidade suscetível, que, aliado à falta de condições ideais de higiene, pode vir a ser um grupo bastante afetado (ABUASSI C e ABUASSI WL, 2006).

$\mathrm{Na}$ análise de 1195 amostras de exames parasitológicos de fezes (EPF), em crianças de 2 a 10 anos, na cidade de Campina Grande - RN, mostrou-se que 553 foram positivos para enteroparasitoses, sendo os mais prevalentes a $E$. coli $(30,9 \%)$ e a $G$. intestinalis $(19,2 \%)$. Em outro estudo, realizado no município de Chapadinha - MA, no qual foram analisadas 3933 amostras de EPF, fora constatado que a faixa etária de 0 a 10 anos e 11 a 20 anos foram as mais acometidas, sendo o complexo E. histolyticalE. díspar e a $G$. intestinalis os protozoários mais frequentes, corroborando com os resultados do estudo atual (SILVA FS, et al., 2010; SILVA MTN, et al., 2014).

Quanto à distribuição dos casos de protozooses positivos classificados por sexo, identificou-se que 1234 participantes são do sexo feminino $(61,76 \%)$, enquanto apenas 764 são do sexo masculino $(38,24 \%)$. Nesse sentido, é importante frisar que a maioria dos estudos que visam analisar a diferença de dados, quanto ao sexo, mostra que as mulheres se previnem mais e buscam com maior frequência as unidades básicas de saúde, além de viverem, em média, 7,3 anos a mais que os homens e morrerem menos de doenças relacionadas à circulação e ao aparelho digestivo (BERTOLINI DNP e SIMONETTI JP, 2014).

Como reforço ao achados acima, o estudo realizado no laboratório de Análises Clínicas do Hospital Universitário Lauro Wanderley (HULW), em João Pessoa-PB, observou que 78\% das amostras positivas para enteroparasitoses eram do sexo feminino. De forma semelhante, nas amostras coprológicas colhidas e analisadas, na unidade de Saúde da Família, no município de Belém - PA, 57,4\% das parasitoses intestinais positivas foram do sexo feminino, contra $42,6 \%$ do sexo masculino (DANTAS SH, et al., 2019; PICANÇO NJA, et al., 2019).

No presente estudo, ocorreu uma discrepância entre a incidência dos pacientes na zona urbana $(67,27 \%)$ e na zona rural (4,35\%). Entretanto, por ser uma enfermidade que afeta, principalmente, as populações residentes em regiões com instalações sanitárias inadequadas, uma justificativa para essa diferença significativa seria uma grande quantidade de subnotificação pela dificuldade ao acesso à saúde pública, substancialmente, para aqueles que moram em regiões da zona rural. No Brasil, sobretudo, em áreas precárias de saneamento básico e com dificuldades ao acesso dos serviços de atenção primária da saúde, temos dificuldades de acesso a dados fidedignos, pois, na maioria dos casos, temos subnotificação (BENCKE A, et al., 2006).

Sabe-se a forte relação entre saneamento básico e parasitoses, sendo a presença dessas doenças um dos fatores mais utilizados para medir a efetividade dos serviços prestados à região. $\mathrm{O}$ alto número de casos e da porcentagem de Entamoeba histolytica, Entamoeba coli e Giardia intestinalis demonstra a ineficiência do saneamento existente em Teresina - PI, que, em 2010, apresentava 61,6\% de domicílios com esgotamento sanitário adequado e, em 2016, teve 1,2 internações por diarreia para cada mil habitantes de acordo com dados do Instituto Brasileiro de Geografia e Estatística (IBGE). Assim, as protozooses intestinais constituem-se doenças que, além de afetarem globalmente a saúde pública, são endêmicas em países em desenvolvimento, atingindo, principalmente, a população com piores condições socioeconômicas.

Tal como exposto anteriormente, a ocorrência de protozooses intestinais promove impactos na qualidade de vida dos indivíduos infectados. Nesse sentido, faz-se necessário que haja investimentos em medidas de controle e prevenção das mesmas, através de pesquisas que caracterizem e identifiquem a prevalência dos casos, direcionando as estratégias e o público-alvo a serem trabalhados de modo a promover a saúde e a prevenir agravos. Em virtude disso, vê-se a importância da realização de estudos que abordem as áreas das zonas rurais do município, já que os dados colhidos deverão permitir a suplementação desse artigo para ajudar nas medidas de prevenção e de controle da doença nas regiões mais prevalentes. 


\section{CONCLUSÃO}

A análise das amostras dos Exames Parasitológicos de Fezes (EPF) sobre a prevalência das protozooses intestinais, durante o período de janeiro de 2018 até setembro de 2018, e a distribuição sociodemográfica dos resultados positivos, permite o entendimento que falta uma infraestrutura no saneamento básico e na conscientização sobre saúde pública na cidade abordada, da mesma forma que ocorre em outras regiões do país. Crianças na faixa etária de 0 a 10 anos e adolescentes de 11 a 20 anos, são os grupos mais expostos a essa enfermidade, podendo trazer prejuízo a longo prazo, como atraso no desenvolvimento neuropsicomotor. Assim, esse artigo visa ampliar o conhecimento sobre o tema, permitindo a suplementação de medidas para a prevenção e o controle das enteroparasitoses, além de ampliar o conhecimento sobre o tema, servindo como base para pesquisas futuras.

\section{REFERÊNCIAS}

1. ABUASSI C, ABUASSI WL. Parasitoses intestinais na adolescência. Adolesc Saude, $2006 ; 3$ (3): $43-46$

2. ANDRADE EC, et al. Parasitoses intestinais: Uma revisão sobre seus aspectos sociais, epidemiológicos e terapêuticos. Rev. APS, 2010; 13 (2): 231-240.

3. BACELAR PAA, et al. Parasitoses intestinais e fatores associados no estado do Piauí: uma revisão integrativa. Revista Eletrônica Acervo Saúde, 2018; 10 (4): 1802-1809.

4. BELO VS, et al. Fatores associados à ocorrência de parasitoses intestinais em uma população de crianças e adolescentes. Rev Paul Pediatr, 2012; 30 (2): 195-201.

5. BENCKE A, et al. Enteroparasitoses em escolares residentes na periferia de Porto Alegre, RS, Brasil. Revista de Patologia Tropical, 2006; 35 (1): 31-36.

6. BERTOLINI DNP, SIMONETTI JP. O gênero masculino e os cuidados de saúde: a experiência de homens de um centro de saúde. Escola Anna Nery Revista de Enfermagem, 2014; 18 (4): 722-727.

7. BISCEGLI TS, et al. Estado nutricional e prevalência de enteroparasitoses em crianças matriculadas em creche. Rev Paul Pediatr, 2009; 27 (3): 289-295.

8. BORGES WF, et al. Parasitos intestinais: elevada prevalência de Giardia lamblia em pacientes atendidos pelo serviço público de saúde da região sudeste de Goiás, Brasil. Revista de Patologia Tropical, 2011; 40 (2): 149-157.

9. BRAGAGNOLLO GR, et al. Intervenção educacional sobre enteroparasitoses: um estudo quase experimental. Revista Cuidarte. 2018; 9 (1): 2030-2044.

10. CARCARÁ MSM, et al. Saneamento básico como dignidade humana: entre o mínimo existencial e a reserva do possível. Eng Sanit Ambient, 2019; 24 (3): 493-500.

11. CHAVES ACP, et al. Revisão do Mecanismo fisiopatológico da amebíase. Revista Augustus, $2010 ; 14$ (29): $74-87$.

12. CRUZ JS, et al. Análise epidemiológica das parasitoses intestinais em escolares de 7 a 12 anos. Revista Eletrônica Acervo Saúde, 2020: 47: e875.

13. DANTAS SH, et al. Perfil socioeconômico e qualidade de vida dos pacientes com protozooses intestinais. Saúde (Santa Maria), 2019; 45 (2): 1-18.

14. DYNIEWICZ AM. Metodologia da pesquisa em saúde para iniciantes. Difusão Editora, 2009.

15. FRANCO RMB. Protozoários de veiculação hídrica: relevância em saúde pública. Rev Panam Infectol, $2007 ; 9$ (1): 36-43.

16. FREITAS MRS, et al. Pesquisa de parasitos intestinais em internatos na cidade de Anápolis-Goiás. Revista Educação em Saúde, 2015; 3 (2): 42-48.

17. GAMBOA MI, et al. Distribution of intestinal parasitoses in relation to environmental and sociocultural parameters in La Plata, Argentina. J Helminthol, 2003; 77 (1): 15-20.

18. HUANG DB, WHITE AC. An Updated Review on Criptosporidium and Giardia. Gastroenterol Clin North Am, 2006 ; 35 : 291-314.

19. IGNACIO CF, et al. Socioenvironmental conditions and intestinal parasitic infections in Brazilian urban slums: a crosssectional study. Revista do Instituto de Medicina Tropical de São Paulo, 2017; 59 (56): 1-10.

20. INSTITUTO BRASILEIRO DE GEOGRAFIA E ESTATÍSTICA (IBGE). Pesquisa Nacional de Saneamento Básico. 2010. Disponível em: <https://cidades.ibge.gov.br/brasil/pi/teresina/panorama>. Acesso em: 10 abr. 2021.

21. LOPES LM, et al. Ocorrência de parasitas e comensais intestinais em crianças da comunidade da Vila Inglesa, em São Paulo, SP, Brasil. Rev Inst Adolfo Lutz, 2010; 69 (2): 252-254.

22. KAPPADOGA S, et al. Antiparasitic Therapy. Mayo Clin Proc, 2011: 86 (6): 561-583.

23. KARANIS P, et al. 2007. Waterborne transmission of protozoan parasites: a worldwide review of outbreaks and lessons learnt. Journal of Water and Health, 2007; 5: 1-38.

24. MAGALHÃES RF. Ocorrência de enteroparasitoses em crianças de creches na região do Vale do Aço - MG, Brasil. UNOPAR Cient. Ciênc. Biol. Saúde. 2013; 15 (3): 187-191.

25. MAIA CVA, HASSUM IC. Parasitoses intestinais e aspectos sociossanitários no nordeste brasileiro no século XXI: uma revisão de literatura. Revista Brasileira de Geografia Médica e da Saúde, 2016; 12 (23): 20-30. 
26. MBUH JV, et al. The incidence, intensity nad host morbidity of human parasitic protozoan infections in gastrointestinal disorder outpatients in Buea Sub division, Cameroom. J Infect Dev Ctries, 2010; 4: 38-43.

27. MONTEIRO ACS. Prevalência e fatores associados à enteroparasitoses em escolares. Dissertação (Mestrado em Modelos de Decisão e Saúde) - Universidade Federal da Paraíba, João Pessoa, 2017; 81 p.

28. NETO AHAM, et al. Uma reflexão sobre as parasitoses intestinais em comunidade de baixa renda do norte do Estado do Ria de Janeiro, Brasil. Revista Práxis, 2009; 1 (2): 71-74.

29. PICANÇO NJA, et al. Prevalência de enteroparasitoses em usuários na faixa etária de 2 a 12 anos atendidos por uma Unidade de Saúde da Família do município de Belém, Pará. Revista Eletrônica Acervo Saúde, 2019: 33: e1321.

30. SANTOS AS, MERLINI LS. Prevalência de enteroparasitoses na população do município de Santa Helena, Paraná. Ciência \& Saúde Coletiva, 2010; 15 (3): 899-905.

31. SANTOS-JUNIOR GO, et al. Prevalência de enteroparasitoses em crianças do sertão baiano pelo método de sedimentação espontânea. Revista de Patologia Tropical, 2006; 35 (3): 233-240.

32. SILVA AMB, et al. Ocorrência de enteroparasitoses em comunidades ribeirinhas do Município de Igarapé Miri, Estado do Pará, Brasil. Revista Pan-Amazônica de Saúde, 2014; 5 (4): 45-51

33. SILVA FS, et al. Frequência de parasitos intestinais no município de Chapadinha, Maranhão, Brasil. Revista Brasileira de Patologia Tropical, 2010; 39 (1): 63-68.

34. SILVA JVL, et al. Factors Associated with Gastrointestinal Parasitic Infections among Young Population in Northeast Brazil. Canadian Journal of Infectious Diseases and Medical Microbiology, 2016; 2016: 1-6.

35. SILVA MTN, et al. Prevalence of entamoeba histolytica/entamoeba dispar in the city of campina grande, in northeastern brazil. Rev. Inst. Med. Trop., 2014; 56 (5): 451-454.

36. VIEIRA PB, et al. Mecanismos específicos de patogenicidade de protozoários de mucosa: Entamoeba hystolitica, Giardia lamblia e Trichomonas vaginalis. Revista HCPA, 2012; 32 (1): 58-70.

37. VIVANCOS V, et al. Giardiasis: Characteristics, Pathogenesis and New Insights About Treatment. Current Topics in Medical Chemistry, 2018; 18: 1287-1303. 